\title{
Online Gait Generation for an Exoskeleton Used in Lower Limb Rehabilitation
}

\author{
Haoping WANG*, Yue YIN \\ Sino-French International Joint Laboratory of Automatic Control and Signal Processing (LaFCAS), \\ School of Automation, Nanjing University of Science \& Technology, Nanjing 210094, China \\ hp.wang@njust.edu.cn (*Corresponding author)
}

\begin{abstract}
Providing walking assistance for mild hemiplegia patients with lower limb rehabilitation exoskeleton has gained considerable attention. The patients' motion characteristics should be emphasized, meanwhile the planning gait should always maintain the balance ability in the walking procedure. In this paper, an online balance gait generation strategy is proposed for an exoskeleton used in lower limb rehabilitation. In the present research, the online gait generation strategy is explained and consists of a gait planning and a gait control strategy. In the gait planning strategy, the healthy swing foot trajectory has been learned, modelled and modified for next cycles' exoskeleton motion by applying improved differential evolution Adaptive Hopf (iDE AHopf) oscillators. Meanwhile to illustrate the human motion intention, the dynamic step length estimation method is utilized to estimate the step length. The discrete step planner determines the motion of the exoskeleton's centre of gravity $(\mathrm{CoG})$. In the gait control strategy, the online balance gait regulation could regulate the exoskeleton joint reference adaptively to confirm the maximum zero-moment-point (ZMP) walking stability according to the PID controller feedback joint signal. Finally, the corresponding simulation results demonstrate the effectiveness of the proposed strategy by verifying the gait learning rate and ZMP stability margin.
\end{abstract}

Keywords: Online gait generation, Lower limb exoskeleton, iDE AHopfoscillators learning, ZMP adaption.

\section{Introduction}

Since the late 1960s, the lower limb exoskeleton has attracted extensive attention all over the world. With the overview of currently available lower limb exoskeletons, the wide application of lower limb exoskeleton is categorized into three types: gait rehabilitation (van Kammen et al., 2016, Zeilig et al., 2012, Simbolotti et al., 2016), walking assistance (Esquenazi et al., 2017, Zhang et al., 2017), and human augmentation (Dumitru et al., 2018).

In the rehabilitation exoskeleton research field, the LOKOMAT (van Kammen et al., 2016), ReWalk (Zeilig et al., 2012), ESKO (Simbolotti et al., 2016) and other medical devices have been designed for gait disorders caused by strokes, brain injuries, or spinal cord injuries in order to enhance their lower extremity gait function. As the current key technology of rehabilitation exoskeleton, the rehabilitation exoskeleton gait research consists of the gait planning and gait controlling fields.

In the gait planning research, one of the common typical methods is inverse kinematics analysis technology. The kinematics analysis technology requires establishing kinematic model and planning swing foot trajectory, the more important is to design CoG motion (Bajrami et al., 2016). To obtain the CoG motion, the cart-table model, the three dimension Linear Inverted Pendulum Model (3D-LIPM) (Kajita et al., 2003, Sato et al., 2011) has been researched firstly, then the liquid model is proposed to simplify the biped exoskeleton (Chafroud et al., 2017).

In order to achieve a stable walking, the ZMP theory (Zhan et al., 2013), which is utilized in biped robot gait generation applications, can be integrated in the walking stability. In (Kajita et al., 2003), the CoG motion is designed for its balance to satisfy the given ZMP reference trajectory using preview control method. It can utilize future ZMP reference to design current $\mathrm{CoG}$ motion in real time. In addition, to search the maximum ZMP margin on the ground, the optimal algorithm e.g. PSO, GA (Fu et al., 2011, Farzadpour \& Danesh, 2012) have been applied into the hip/knee joint motion using iterative calculation.

To maintain the optimal ZMP stability, especially facing the unexpected walking surroundings, some solution methods are regulating the foot landing position, adjusting the ankle joint torque and compensating the joint motion (Zhan et al., 2013, Ding et al., 2015, Sato et al., 2008), and these have achieved better applications.

In order to describe the patients walking characteristics and estimate his/her motion intention, the human-data capturing and learning technology attracts more interests from experts in gait planning research (Cannell et al., 2018), and the technology requires collecting and learning normal human motions. 
For mild hemiplegia patients, one leg has normal walking ability and the other leg has walking dysfunction. To solve this, the online trajectory generation method that can be applied for hemiparetic subjects has been proposed (Vallery et al., 2009). The desired states for one disabled leg are generated online based on the other healthy leg's movements.

Central pattern generators (CPGs) learning as one of gait learning technologies becomes gradually a hot research field (Righetti \& Ijspeert, 2006). The biological neural networks, CPGs, have been shown to produce rhythmic outputs. Using the CPGs model like Rayleigh Oscillators to generate the locomotion for biped robot has been successfully achieved (Mondal et al., 2011). In addition, Hopf oscillator as one of the classical nonlinear CPGs is studied in (Abusabah et al., 2017). It is the biological neural network that autonomously produces rhythmic patterned outputs without sensory feedback, which are increasingly used to control the locomotion of humanoid robots, especially in online generating periodical motion. It has the ability to dynamically alter the amplitude and frequency of oscillator to learn any periodic input signal. In (Liu et al., 2017), Adaptive Hopf oscillators learning strategy is utilized to model the exoskeleton knee motion trajectory by tuning its frequency and amplitude. In addition, the swinging foot trajectory motion as the human walking characteristic has also been researched. The normal individual swing foot trajectories have been learned and modeled by Dynamic Moment Primitives (DMPs) oscillators (Kulvicius et al., 2013) to generate walking gait for the lower limb exoskeleton. In fact, the exoskeleton joint motion generated by oscillators attracts a lot attention from world (Chen et al., 2018).

The existed physical Human Robot Interaction (pHRI) could cause the patients' gait and the exoskeleton's gait different. The essential pHRI force signal (Huang et al., 2018, Long et al., 2018) has been used to generate gait pattern as the motion intensions. In (Long et al., 2018), the pHRI force signal has been observed, and the increment of the angular position of the knee joints has been adjusted in real time. The online sparse Gaussian process algorithm is proposed to learn the human gait trajectory in order to ensure that the exoskeleton system shadows the human gait trajectory in the robotic gait rehabilitation systems. In (Chen et al., 2017), the patients' CoG motion is the intension to adjust exoskeletons' CoG motion. Thus, in the walking assistance procedure, the exoskeleton can utilize pHRI and adapt to the patients' motion intension is vital to improve patients' motion comfort (Hamaya et al., 2017).

In the gait controlling research, the main methods focus on the planning joint position tracking strategy. Considering the complex walking surrounding and high-order nonlinear model, the model free controller (MFC) has been developed and implemented with a high precision control mainly based on the input-output (I/O) data (Wang et al., 2016, Han et al., 2018). A model-free (MFC) based adaptive nonsingular fast terminal sliding mode control strategy is proposed in (Han et al., 2018) in order to speed up the convergence rate and to improve the tracking performance. However, the lower limb exoskeleton controller only tracks the desired pre-defined trajectory rather than adapting it to the controller error, which means to adjust the desired gait reference real time.

Therefore, how to achieve efficient exoskeleton planning gaits which satisfy the patients' different gait characteristic, satisfy their different motion intensions, and generate stable walking patterns, are urgent problems needed to be solved in this paper. These are closely related to lower limb rehabilitation effects (Hamaya et al., 2017).

To overcome these problems, the online balance gait generation strategies are proposed for an exoskeleton used in lower limb rehabilitation. It could utilize the healthy limb of a mild hemiplegia patient as the gait characteristic to tune the exoskeleton gait, also it could achieve a better balance performance. This entire method consists of gait planning strategy and gait control strategy. In the gait planning strategy structure, the iDE AHopf oscillators have been used to learn, generate and modify the swing foot motion trajectories and apply them into assistance walking. Then, in order to adapt it to the patient, the next step length has been determined by the step length adaption method

https://www.sic.ici.ro 
according to the patients' motion intension. The discrete step planner designs the exoskeleton $\mathrm{CoG}$ motion. In the gait control strategy structure, the joint controller has been designed to track the joint trajectory obtained from inverse kinematic models. The balance gait adaptive regulation technology has been researched in the humanexoskeleton model to improve the dynamic balance ability as well.

The rest of this paper is organized as follows. The rehabilitation exoskeleton description and modeling structure are introduced in section II. In section III, the online dynamic gait generation strategy which is based on iDE AHopf oscillators is derived. Section IV gives the proposed method's simulation results. Finally, conclusions and perspectives are shown in section $\mathrm{V}$.

\section{Exoskeleton Description and Modelling}

The whole rehabilitation exoskeleton walking procedure is made of a single-support phase (SSP) and a double-support phase (DSP). It is a 3-D biped multi-mass CoG model with a serial link structure as shown in Figure 1. Figure 2 shows the exoskeleton SimMechanic platform structure. Table 1 provides the mechanical structure of the parameters of link length and mass. The exoskeleton contains two degrees of freedom (DOFs) in a coronal plane, two DOFs in a transverse plane and six DOFs e.g. hip, knee and ankle joint of double legs in a sagittal plane. Figure 1 depicts that the origin of coordinate is set on the contacted ground of the support leg. The $\mathrm{x}$-axis is the forward direction, the $y$-axis is the horizontal direction, and $z$-axis is the vertical direction.

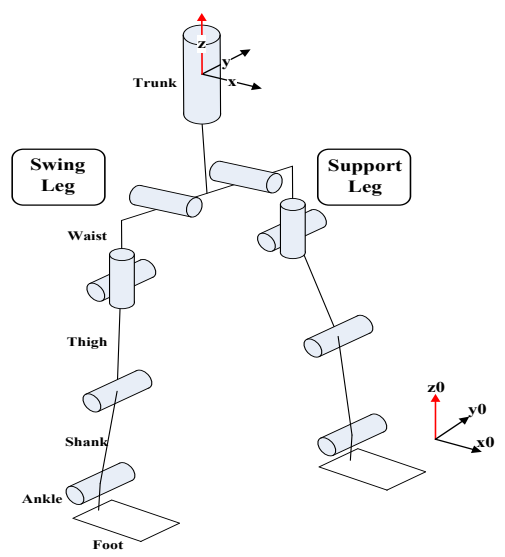

Figure 1. Three-Dimension exoskeleton model

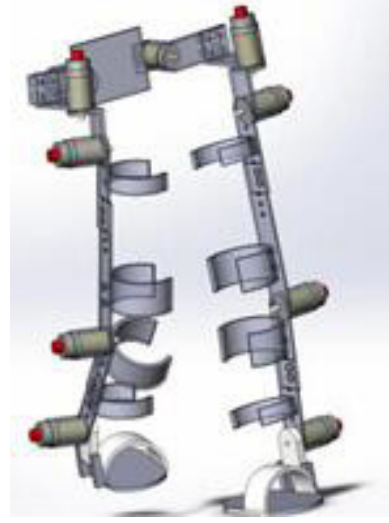

Figure 2. SimMechanic platform of lower Limb exoskeleton

Table 1. Parameters of link length and mass

\begin{tabular}{ccc}
\hline Link Structure & Length $(\mathrm{m})$ & Mass $(\mathrm{kg})$ \\
\hline Trunk & 0.85 & 10 \\
Waist & 0.019 & 0.154 \\
Thigh & 0.52 & 7 \\
Shank & 0.4 & 5.255 \\
Ankle & 0.0 & 0 \\
Foot & 0.282 & 2.5 \\
\hline
\end{tabular}

Here only the ankle, knee and hip joint are considered, and the other DOFs were set to zero. According to the Denavit-Hartenberg (D-H) theory, the exoskeleton forward kinematic model is built at each DOF from the support foot to swing foot as follows:

$T=\left[\begin{array}{cccc}-1 & 0 & 0 & a_{5} c_{1}+a_{4} c_{3}-a_{4} c_{8}-a_{5} c_{10}-2 a_{1} s_{5} \\ s_{4} s_{7} & -c_{47} & -s_{47} & 2 a_{2} s_{4}+a_{5} s_{1}-a_{4} s_{3}+2 a_{1} c_{5}-a_{4} c_{47} s_{8}+a_{5} c_{47} s_{10} \\ -c_{4} s_{7} & -s_{47} & c_{47} & a_{5} s_{47} s_{10}-2 a_{1} c_{4} c_{5}-a_{4} s_{47} s_{8}-2 a_{2} c_{4} \\ 0 & 0 & 0 & 1\end{array}\right]$

where the applied notations are defined as $S_{i}=\sin \theta_{i}, C_{i}=\cos \theta_{i}, S_{i q}=\sin \left(\theta_{i}+\theta_{q}\right), C_{i q}=\cos \left(\theta_{i}+\theta_{q}\right)$. Given the walking $\mathrm{CoG}$ motion trajectory and terminate foot trajectory in the cartesian coordinate system, the joint degree trajectory can be calculated i.e. Inverse Kinematics. The steps which illustrate the inverse kinematics are given below. First, from support foot and the $\mathrm{CoG}$ coordinates, the support leg kinematic model can be obtained

${ }^{\text {sup }} A_{0}^{5}=A_{0}^{1} A_{1}^{2} A_{2}^{3} A_{3}^{4} A_{4}^{5}$

where $A_{i}^{j}$ represents the transformation matrix from $i$ th joint to $i$ - 1 th joint coordinate. Then using the kinematic matrix inversing method, the knee 
and hip joint solutions of support leg $\theta_{s p k}, \theta_{s p h}$ respectively are shown as

$\theta_{s p k}=-\arccos \frac{\left(a_{2} s_{4}-p_{y}\right)^{2}+\left(p_{x}-a_{3}\right)^{2}-a_{5}^{2}-a_{4}^{2}}{2 a_{4} a_{5}}$

$\theta_{s p h}=\arctan \frac{a L_{2}-b L_{1}}{a L_{1}+b L_{2}}$

with

$a=p_{x}-a_{3}, b=p_{y}-a_{2} s_{4}, L_{1}=a_{4}+a_{5} c_{2}, L_{2}=-a_{5} s_{2}$

Assuming the foot parallel to the ground always, the support ankle joint $\theta_{s p a}$ could be obtained.

$\theta_{s p a}=-\theta_{s p h}-\theta_{s p k}$

Next, according the terminate foot trajectory in the cartesian coordinate system and the parallel foot constraint, the swing leg knee, hip and ankle joint $\theta_{s w k}, \theta_{s w h}$ and $\theta_{s w a}$ are obtained as well.

$$
\begin{aligned}
\theta_{s w k} & =-\arccos \left[\frac{\left(s_{7}\left(a_{2}+p_{z e} c_{6}+p_{x e} s_{6}\right)+p_{y e} c_{7}\right)^{2}}{2 a_{4} a_{5}}\right. \\
& \left.+\frac{\left(a_{3}+p_{x e} c_{6}-p_{z e} s_{6}\right)^{2}-a_{4}^{2}-a_{5}^{2}}{2 a_{4} a_{5}}\right] \\
\theta_{s w h} & =\arctan \frac{a L_{2}+b L_{1}}{-a L_{1}+b L_{2}} \\
\theta_{s w a} & =-\theta_{s w k}-\theta_{s w h}
\end{aligned}
$$

with

$a=a_{3}+p_{x e} c_{6}-p_{z e} s_{6}, b=-a_{2} s_{7}-p_{x e} s_{6} s_{7}-p_{y e} c_{7}-p_{z e} c_{6} s_{7}$, $L_{1}=a_{4}+a_{5} c_{9}, L_{2}=a_{3} s_{9}$.

So far, the forward kinematics and inverse kinematics analysis are completed.

\section{Online Balance Gait Generation Strategy}

The online balance gait generation strategy is to enable the patient to acquire and model the healthy swing foot trajectory as walking characteristics utilized to tune the exoskeleton. It could implement balance walking patterns based on ZMP adaption theory. The online balance gait generation framework shown in Figure 3 is proposed to generate the exoskeleton motion trajectories.

This proposed framework consists of the gait planning strategy and gait control strategy. In the gait planning strategy, the exoskeleton could learn the mild hemiplegia patients' healthy limb motion online. In previous walking cycles, the swing foot trajectory from the patient healthy limb motion is collected and learned, and then it is modeled by the iDE AHopf oscillators. The output of iDE AHopf oscillators could be modified as the reference exoskeleton swing foot trajectory in Cartesian space for current and next walking cycles. Additionally, the motion intention is estimated to adapt the step length $S$, and then the discrete step planner can generate continuous $\mathrm{CoG}$ motion according to dynamic estimated $S$.

In the gait control strategy, the swing foot trajectory and CoG motion trajectory in Cartesian space have been turned into the exoskeleton reference joint trajectory by inverse kinematic. The PID controller is used to track motion, and the real exoskeleton feedback from exoskeleton sensor data is utilized to adapt the gait online and to improve the balance walking stability by using ZMP compensation theory, which is defined as balance gait adaptive regulation in this paper. More details about the gait planning strategy and gait control strategy are illustrated in the following subsections.

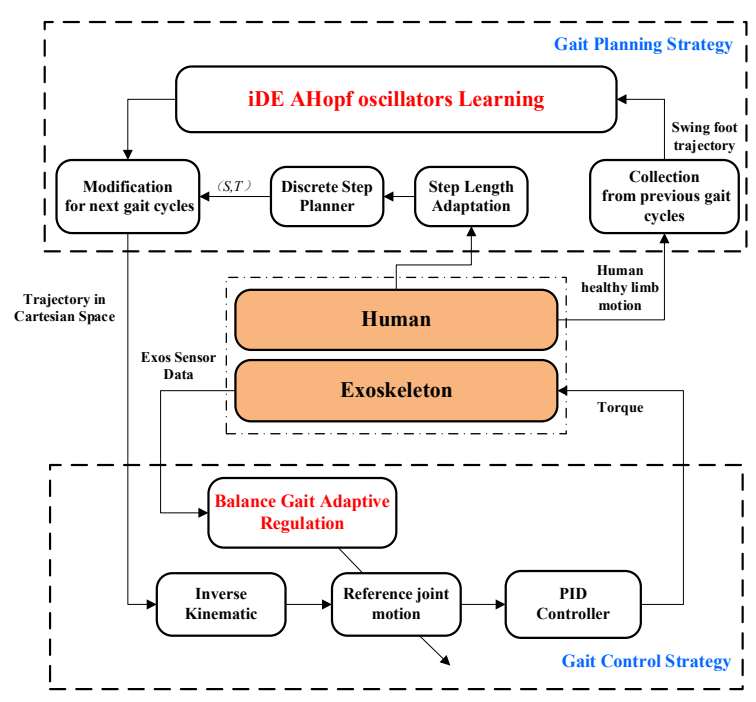

Figure 3. Online balance generation framework

\subsection{Gait Planning Strategy Design}

In this research, the swing foot trajectory are learned, modeled and modified based on iDE AHopf oscillators. After obtaining the estimated step length $S$, the CoG motion was planned based on cart-table model. Thus this gait planning strategy consists of the foot trajectory learning based on iDE AHopf oscillators, the step length adaption and the discrete step planner employed to generate $\mathrm{CoG}$ motion. 


\subsubsection{Foot Trajectory Learning Based on iDE AHopf Oscillators}

In the procedure of learning periodical signals, the AHopf oscillators parameters, coupling driving constant and time constant $\tau$ respectively, could describe the strength of coupling between the oscillator and mechanical system, which influence the converge rate and learning error in AHopf oscillators learning method.

Hence in this section, the iDE AHopf oscillators learning structure is proposed to learn and generate the gait motion, which is used to learn and model the swing foot trajectory of a mild hemiplegia patient's healthy limb. The iDE algorithm is utilized to regulate the optimal AHopf oscillators' parameters to describe the actual complex varying human-exoskeleton interaction system.

In order to determine the parameters, the reference periodical signals have been collected exoskeleton motion trajectories, which are measured from the patients healthy limb motion in advance, and the iDE algorithm evaluates on the learning error and searches on optimal parameters for AHopf oscillators. And by sufficient iteration, its obtained parameters are able to describe actual human interaction motion better, which could achieve a fast learning rate for the desired reference trajectory.

The structure of this system is illustrated in Figure 4. From Figure 4, the obtained optimal AHopf oscillators' parameters would be used in the swing foot trajectory learning, modification and generation.

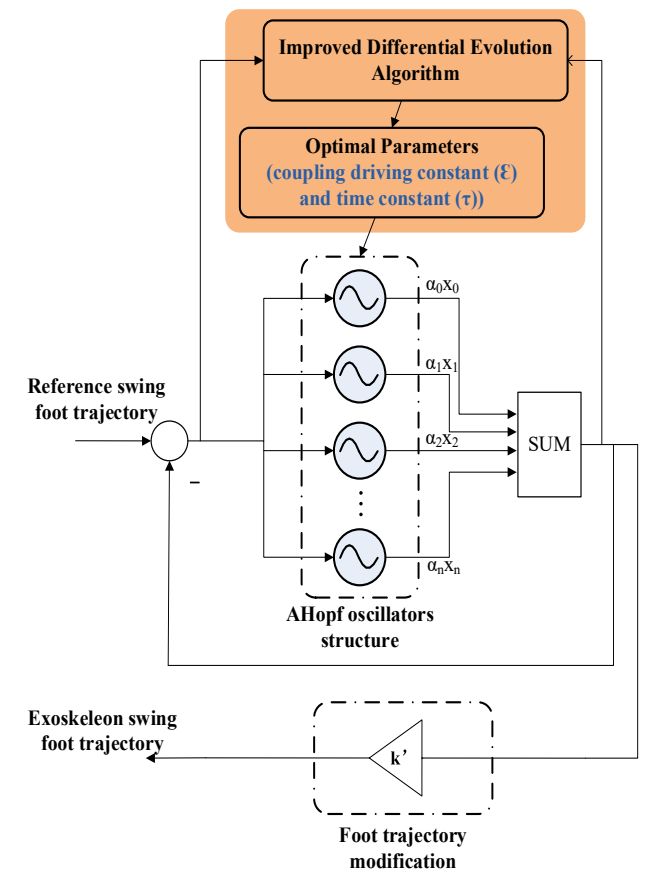

Figure 4. The structure of iDE AHopf oscillators
To illustrate the learning structure, the section is divided into two parts: AHopf oscillators learning structure and iDE algorithm.

\subsubsection{Adaptive Hopf (AHopf) Oscillators Learning Architecture}

This AHopf oscillators learning architecture is made of coupled adaptive frequency Hopf oscillators as shown in Figure 4. Each Hopf oscillator (Righetti et al., 2006), is described as follows:

$\dot{r}=\gamma\left(\mu-r^{2}\right) r+\varepsilon F \cos \phi$

$\dot{\phi}=w-\frac{\varepsilon}{r} F \sin \phi$

$\dot{w}=-\tau F \sin \phi$

where $r$ is the oscillators system parameters, $\phi$ represents the phase of oscillators, $\mu$ represents the amplitudes of oscillation and can be set to $1, \gamma$ controls the speed of recovery perturbation, $w$ controls the frequency of oscillation, $F$ is the periodic driving signal which motivates each Hopf oscillator to alter itself, $\varepsilon$ is the strength of coupling between the oscillator and mechanical system, and $\tau$ is the time constant.

The dynamic $F$ drives each Hopf oscillator to modify its frequency and motion trajectory, and each Hopf oscillator motion is considered a weighted sum (Liu et al., 2017) as shown in (11), The learning output data, i.e. the interacted foot trajectory motion, can then be obtained.

$\theta_{\text {learn }}(t)=\sum_{i=0}^{N} \alpha_{i} x_{i}=\sum_{i=0}^{N} \alpha_{i} r_{i} \cos \phi_{i}$

where $\alpha_{i}$ represents the gain associated to the frequency $\omega_{i}$ of oscillator $i, N$ means the number of oscillators, here set to 5 . Let $F(t)$ represent the negative feedback corresponding to the teaching signals $\theta_{\text {refer }}(t)$, while $\eta$ represents the learning rate for amplitude $\alpha_{i}$.

$$
\begin{aligned}
& F(t)=\theta_{\text {learn }}(t)-\theta_{\text {refer }}(t) \\
& \dot{\alpha}_{i}=\eta r_{i} \cos \phi_{i} F(t)
\end{aligned}
$$

From formula (8) to (13), the AHopf oscillators could achieve learning trajectory signals autonomously.

\subsubsection{Improved Differential Evolution (iDE) Algorithm}

The improved differential evolution (iDE) algorithm has been embedded into AHopf 
oscillators to search the optimal AHopf oscillators' parameters by iterative learning in order to ensure a fast convergence to the desired signals.

The scheme of the iDE AHopf oscillators learning algorithm is shown in Figure 5. Compared to DE (Qin et al., 2009), an adaptive mutation with Levy flight search algorithm was utilized in the mutation operation, which was defined as the iDE algorithm. It would start with arbitrarily generated and evenly distributed initial population. Subsequently it repeatedly applies ITASE error evaluations, adaptive mutation with Levy flight search algorithm, crossover and selection operations to keep the best AHopf oscillators parameters.

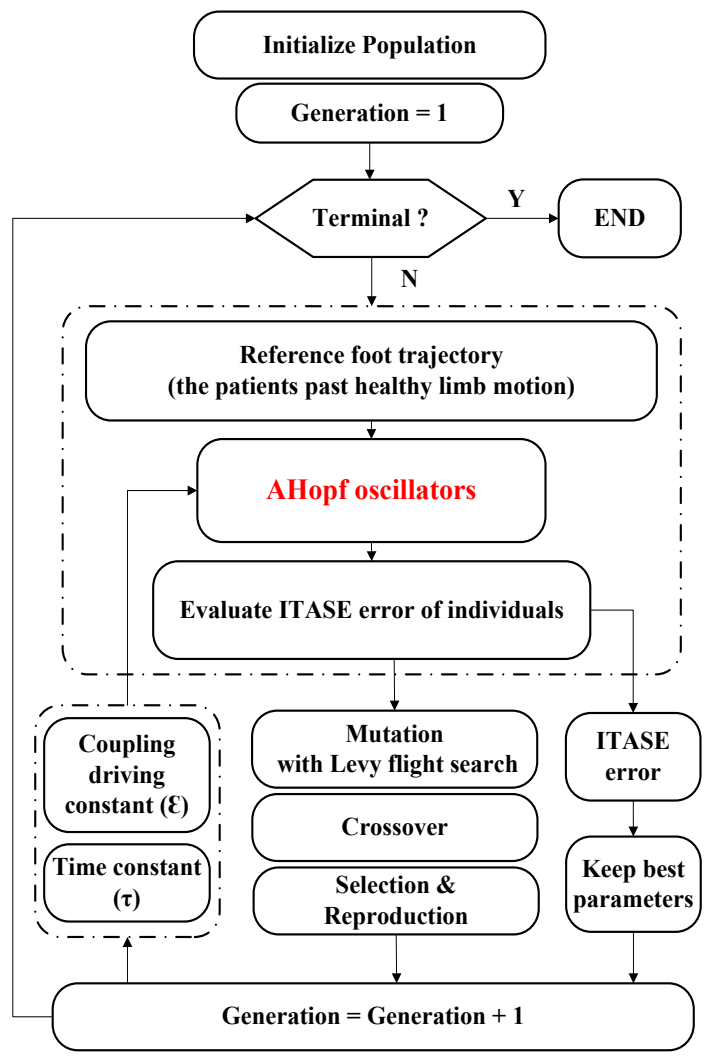

Figure 5. The scheme of iDE AHopf oscillators learning algorithm

Here, the iDE search parameter achieves the minimum of Integrated Time, Absolute and Square Error (ITASE) fitness function (14).

ITASE $=\lambda \int_{0}^{\infty} t|e(t)| d t+(1-\lambda) \int_{0}^{\infty} t e^{2}(t) d t$

where $e(t)=y(t)-y_{0}(t), y(t)$ is the output signal, and $y_{0}(t)$ is the reference signal, $\lambda$ is the weighted constant, which has been set to 0.5 . The reference signal has been set to the patients past healthy limb motion periodically. The iDE searched parameters are accurate and practical, which could learn the patient's limb motion rapidly.
During population mutation, iDE employs the process of creating a mutant vector $v_{i}(t)$ from parent vector $x_{i}(t)$ using mutation operator. The scaling factor $F$ is regulated adaptively, and the levy flight search algorithm in (Kumar et al., 2015), a local search approach, is utilized to search for the global optimum, to avoid the local optimum of search solution space, and to maintain the proper balance between exploration and exploitation behavior of conventional DE.

The mutation operation with Levy flight search algorithm is defined as follow:

$v_{i}(t)=x_{b}(t)+F(t) \times\left(x_{m}(t)-x_{w}(t)\right)+s s(t) \times\left(x_{m}(t)-x_{w}(t)\right)$

where $F(t)$ represents the adaptive scaling factor and $s s(t)$ represents the levy flight step size.

Here the $F$ adaptive regulation is defined as:

$F(t)=F_{l}+\left(F_{u}-F_{l}\right) \frac{f_{m}-f_{b}}{f_{w}-f_{b}}$

where $F_{l}=0.1, F_{u}=0.9$, and $f_{b}, f_{m}$ and $f_{w}$ represent the ITASE fitness of the best one $x_{b}(t)$, the medium one $x_{m}(t)$ and the worst one $x_{w}(t)$ from three selected mutant vectors respectively.

Additionally, the local search strategy which is inspired by Levy flight random walk has been proposed. The walk steps are defined in terms of the step-lengths, which have a certain probability distribution. The levy flight step size has been generated in order to exploit the search area based on Mantegna's algorithm for levy stable distribution as shown in formula (17).

$s s(t)=0.005 \times s(t) \times S L C$

where $s s(t)$ represents the levy flight step size, which has been established by social learning component $(S L C)$ of the global search algorithm and 0.005 represents the multiplier utilized to maintain the solution within the system boundary, and $s(t)$ is defined as follows:

$s(t)=\frac{u}{|v| \frac{1}{\beta}}$

where $u, v$ are derived from normal distributions: $u \sim N\left(0, \sigma_{u}^{2}\right), v \sim N\left(0, \sigma_{v}^{2}\right)$, and $\beta$ is the constant used to calculate the normal distributions in variance (Kumar et al., 2015), which has been set to 1.5 defined as follows: 
$\sigma_{u}=\left[\frac{\Gamma(1+\beta) \cdot \sin \left(\frac{\pi \beta}{2}\right)}{\beta \cdot \Gamma\left(\frac{1+\beta}{2}\right) \cdot 2^{\frac{\beta-1}{2}}}\right]^{\frac{1}{\beta}}, \sigma_{v}=1, \quad \Gamma(1+\beta)=\int_{0}^{\infty} t^{\beta} e^{-t} d t$

Here $\Gamma($.$) is the Gamma function, then using$ crossover and selection operation, the best solution for the next iteration has been chosen.

Obtained the best parameters using the iDE algorithm, the AHopf oscillators are used to learn previous swing foot trajectory of the healthy limb, the gain $\alpha_{i}$ can be adaptively adjusted, associated to the frequency $\omega_{i}$ of oscillator $i$. The gain $\alpha_{i}$ and the frequency $\omega_{i}$ can be also adaptively adjusted according the next excepted step length $S$ and gait cycle period $T$ respectively.

Here two AHopf oscillators have been used in order to learn and model the foot motion in forward $(X)$ and vertical $(Z)$ direction. The original foot trajectory is obtained by using the Kinect capture method in (Seleem \& Assal, 2016). In order to describe the different swing foot motion with $S$, it means to modify the gain $k^{\prime}$. The output of AHopf oscillators $\hat{\theta}_{\text {learn }}(t)$ was multiplied by the gain $k$ ' to adjust the swing foot motion in forward direction as follows:

$$
\hat{\theta}_{\text {final }}(t)=k^{\prime} \theta_{\text {learn }}(t)=k^{\prime} \sum_{i=0}^{N} \alpha_{i} r_{i} \cos \phi_{i}
$$

The trajectory results are shown in Figure 6, which illustrates the swing foot trajectory in Cartesian space according to the variable gain $k^{\prime}$ as $0.8,0.9$, $1.0,1.1,1.2$ with respect to step length $S$ of 0.3 , $0.35,0.4,0.45$ and $0.5 \mathrm{~m}$ respectively.

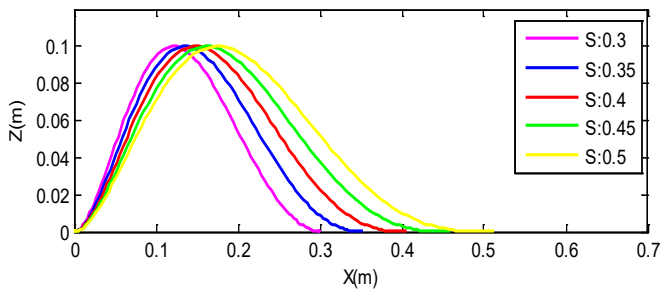

Figure 6. Foot trajectories with different parameters $S$ based on iDE AHopf oscillators

\subsubsection{Step Length Adaption}

In this sub-section, the adaptive step length estimation method is employed to determine the step length adaption in the DSP and plan a gait at the beginning of each step according to the patients' motion intention. In (Chen et al., 2017), the relationship between the human $\mathrm{CoG}$ position and support polygon length in sagittal plane has been estimated in order to correct the next step length utilized to implement the human dynamic balance gait.

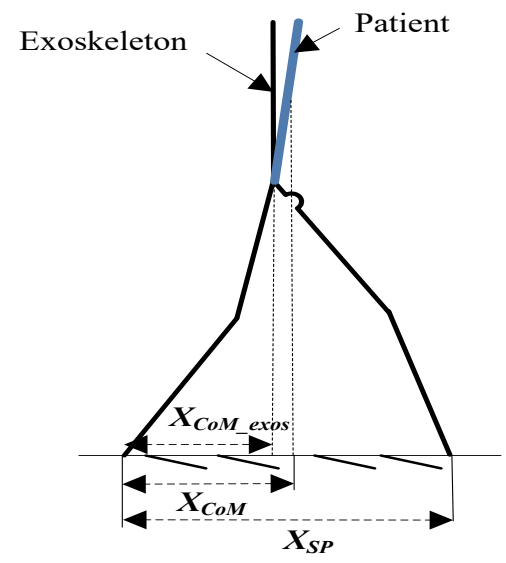

Figure 7. The relationship between human $\mathrm{CoG}$ position and support polygon

Here, as shown in Figure 7, the estimated goal $S$ used to correct online the length of each step due to the patients' motion intention which is defined as follows:

$S_{i}=S_{i-1}+\alpha\left(\hat{X}_{C o G}-\frac{X_{C o G}}{X_{S P}}\right)$

where $i$ is the step index, $S_{i-1}$ and $S_{i}$ are the $i-1$ th and $i$ th step length respectively, and $\alpha$ is the estimation parameter, here is set at $-0.25 . X_{S P}$ represents the support polygon which equals to $S_{i-1}, X_{C o G}$ represents the human CoG position. Here one defines:

$\hat{X}_{C o G}=\frac{X_{\text {CoG_exos }}}{S_{i-1}}$

where $X_{C O G \text { exos }}$ represents the exoskeleton CoG position. The distance $\Delta$ between $X_{C o G}$ and $X_{\text {CoG_exos }}$ could be obtained as follows:

$\Delta=X_{C o G}-X_{\text {CoG_exos }}$

The exoskeleton could adjust its step length according to upper body CoG position $X_{C O G}$ and $\Delta$. Once the human $\mathrm{CoG}$ position exceeds the exoskeleton CoG position, the step length $S$ should increase. By contrast, the step length $S$ decreases.

And next, foot trajectory could be modified based on learned iDE AHopf oscillators according the adaptive step length $S$. 


\subsubsection{Discrete Step Planner}

After dynamically obtaining the step length and the swing foot trajectory with AHopf oscillators, the future ZMP reference $p^{\text {ref }}$ has been planned, and the discrete step planner has been designed to generate the next CoG motion at instantaneous double-support phase (DSP) according the next foot position and current CoG position, velocity and acceleration. In this section, the ZMP theory has been applied into the exoskeleton $\mathrm{CoG}$ generation. The complicated human and exoskeleton system model can be simplified as cart-table model, and the following ZMP equation representing the relationship between CoG and ZMP (Kajita et al., 2003) can be obtained.

$$
p_{x c}=x-\frac{z_{c}}{g} \ddot{x}
$$

where $p_{x c}$ is the ZMP location of cart-table model on the floor around $\mathrm{x}$-axis i.e. forward direction, $x$ represents the $\mathrm{CoG}$ position in $\mathrm{x}$-axis, $z_{c}$ is the model COG height, and $g$ is the gravity acceleration.

Here the optimal preview controller could utilize the future ZMP information to design the current smooth CoG motion, which has been introduced in this paper (Wu et al., 2013).

\subsection{Gait Control Strategy Structure}

From the gait plan strategy structure, the swing foot trajectory modeled by iDE AHopf oscillators and CoG motion can be obtained, Then, the reference joint trajectory $\theta^{*}$ is obtained by using its corresponding inverse kinematic transformation.

Considering the multibody exoskeleton model in Figure 1, the simplified cart-table model could barely describe the real ZMP stability. Additionally, the exoskeleton might not implement the planning gait tracking performance due to the external disturbance from ground and the PID joint controller error, which can cause out range of walking stable region. Each joint reference should be regulated to achieve superior balance gait. The proposed gait control strategy is shown in Figure 8.

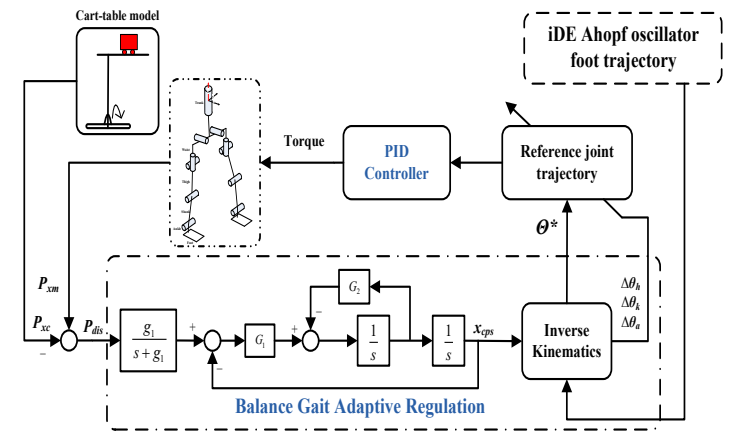

Figure 8. The gait control Strategy Structure

The balancing gait adaptive regulation approach from (Sato et al., 2008) is utilized. It focuses on improving the exoskeleton system gait stability with respect to the current joint controlling state. It can also calculate the walking ZMP real time, and modify each joint position and velocity simultaneously according exoskeleton feedback gait in order to improve the maximum of ZMP stable margin. The real joint position could be detected by encoder, and the current real ZMP $p_{x m}$ could be calculated using formula (25).

$p_{x m}=\frac{\sum_{i=1}^{N} m_{i}\left[\left(\ddot{z}_{i}+g\right) x_{i}-z_{i} \ddot{x}_{i}\right]}{\sum_{i=1}^{N} m_{i}\left(\ddot{z}_{i}+g\right)}$

where $p_{x m}$ represents the multibody exoskeleton ZMP response, $m_{i}$ is the mass corresponding to link $i, x_{i}$ and $\ddot{x}_{i}$ are the center position and acceleration of $i$ th link in forward direction respectively, $z_{i}$ and $\ddot{z}_{i}$ are the center position and acceleration of $i$ th link in vertical direction, $g$ is the gravity acceleration.

As seen in Figure 8, the multibody exoskeleton ZMP tracking error $p_{d i s}$ is obtained by the difference between the cart-table model ZMP in formula (24) and the current real exoskeleton ZMP in formula (25), Then $p_{d i s}$ is low-pass filtered, and the position compensation is implemented. Here $g_{1}=4.01, G_{1}=1, G_{2}=2$.

Besides, the external CoG motion bias $x_{c p s}$ is calculated directly to overcome the sudden ground disturbance. Using inverse kinematic function $f\left(x_{c p s}\right)$, the hip, knee and ankle joint increments $\left(\Delta \theta_{h}, \Delta \theta_{k}, \Delta \theta_{a}\right)$ have been calculated to improve ZMP tracking ability.

\section{Simulation and Results}

In this section, the $0 \sim 12 \mathrm{~s}$ walking procedure is designed in MATLAB/Simulink and SimMechanics 
simulation platform. The gait cycle period has been set to $2 \mathrm{~s}$; the initial step length has been set at $0.32 \mathrm{~m}$. The swing foot trajectory was collected from the normal individual gait in advance. The collected periodical trajectory has been used to imitate the healthy limb motion for gait generation.

Using the online balance gait generation strategy, the swing foot trajectory could be modeled by iDE AHopf oscillators; the CoG motion could be planned by discrete step planner, and the step length could be estimated. Finally, to evaluate the proposed strategy, the gait generation results and the ZMP stability of the generated gait are taken into consideration.

\subsection{Gait Generation Results}

These results include the swing foot trajectory learning, step length adaption and online gait generation results.

\subsection{1 iDE AHopf Oscillators Learning Comparison}

In the present method, the swing foot trajectory in both vertical and forward direction has been learned based on the iDE AHopf oscillators learning in order to search the optimal coupling parameters. Here the method using DE algorithm to optimize the AHopf oscillators is named DE AHopf oscillators learning algorithm, which is compared with iDE AHopf oscillators learning.

The iteration number has been set as 150 times, the ITASE has been defined as the square sum of ITASE value in forward direction and vertical direction as seen in Figure 9. It can be observed that iDE can search for the parameters of the AHopft oscillator more rapidly, which demonstrates that the superior performance of iDE AHopf oscillators learning algorithm.

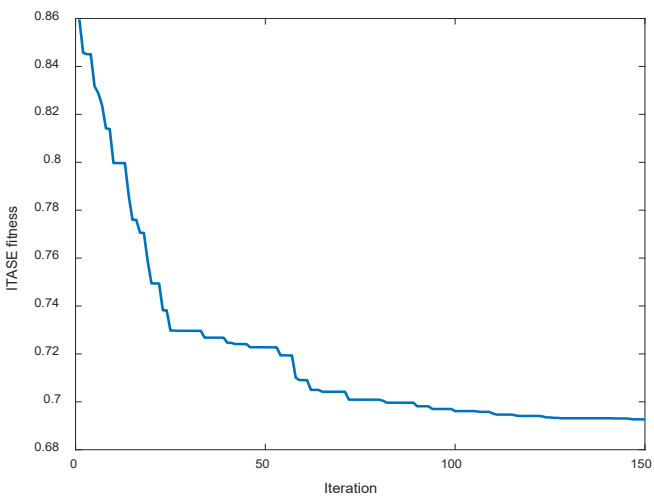

(a) DE AHopf oscillators learning iteration

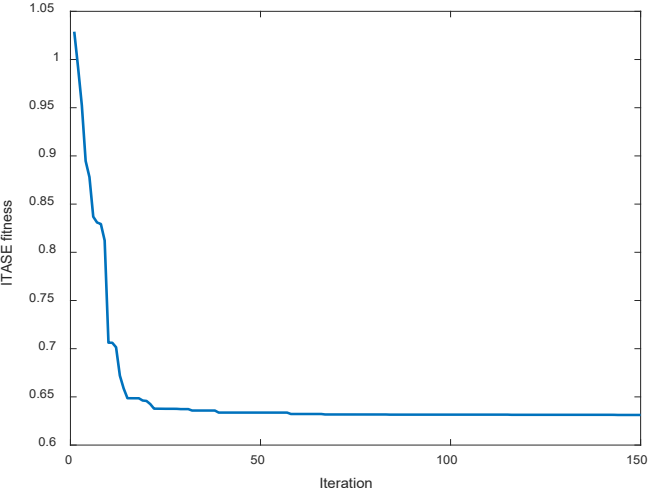

(b) iDE AHopf oscillators learning iteration

Figure 9. Oscillators learning iteration comparison

Here, the performance of iDE AHopf oscillators has been verified by comparing the AHopf oscillators shown in Figure 10. From Figure 10, it can be noticed that by comparing DE AHopf oscillators learning algorithms, the swing foot trajectory could rapidly converge to the desired motion signal in one gait cycle, and the learning error becomes small enough.

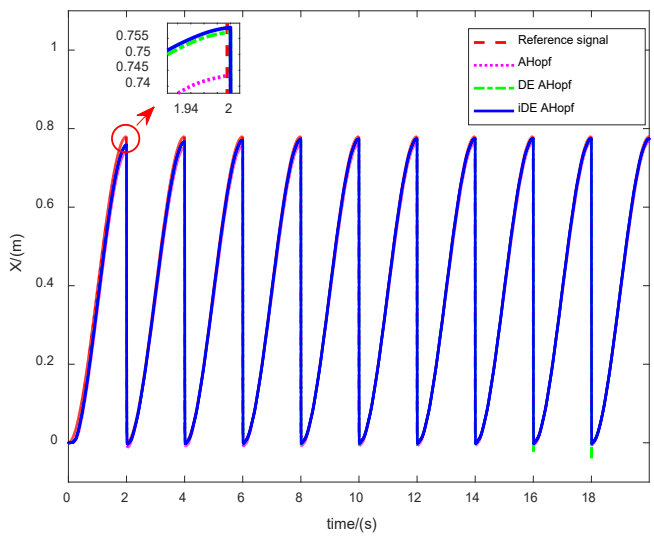

(a) Forward direction

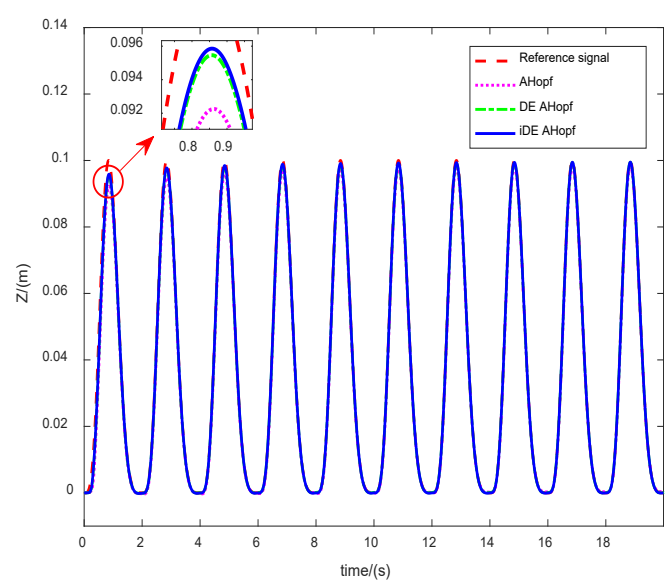

(b) Vertical direction

Figure 10. Foot trajectory learning result comparison 
Besides, to evaluate the effectiveness of iDE AHopf oscillators learning methods, the ITASE error results calculated in formula (15) have been compared as shown in Table 2. It can be observed that the ITASE value of iDE AHopf oscillators learning is smaller than AHopf and DE AHopf oscillators learning error.

Table 2. Learning error comparing results

\begin{tabular}{ccc}
\hline $\begin{array}{c}\text { Compared } \\
\text { methods }\end{array}$ & $\begin{array}{c}\text { ITASE } \\
\text { (forward direction) }\end{array}$ & $\begin{array}{c}\text { ITASE } \\
\text { (vertical direction) }\end{array}$ \\
\hline AHopf & 3.7814 & 2.3741 \\
DE AHopf & 2.1077 & 0.7809 \\
iDE AHopf & 1.8914 & 0.5231 \\
\hline
\end{tabular}

\subsubsection{Step Length Adaption Result}

After 6 s, the step length $\mathrm{S}$ adapts to the human $\mathrm{CoG}$ position, the difference $\Delta$ between exoskeleton $\mathrm{CoG}$ position and human $\mathrm{CoG}$ position causes step length adaption, $\Delta$ is positive which means that $S$ would increase, and $\Delta$ is positive which means that the $S$ would decrease as shown in Table 3.

Table 3. The results of each step length

\begin{tabular}{ccc}
\hline Step Number $i$ & $S_{i}$ & $\Delta$ \\
\hline 1 & 0 & $\backslash$ \\
2 & 0.32 & $\backslash$ \\
3 & 0.32 & 0.0156 \\
4 & 0.3322 & -0.0464 \\
5 & 0.2973 & 0.0349 \\
6 & 0.3266 & -0.0234 \\
7 & 0.3087 & 0.0030 \\
\hline
\end{tabular}

\subsubsection{Online Gait Generation Result}

By obtaining the estimated step length $S$, the collected and modeled healthy limb's swing foot trajectory would be modified with iDE AHopf oscillators for inverse kinematic analysis, which could adapt to the patient's walking requirements and characteristics.

Firstly, the swing foot trajectory modified with iDE AHopf oscillators is given in Figure 11.

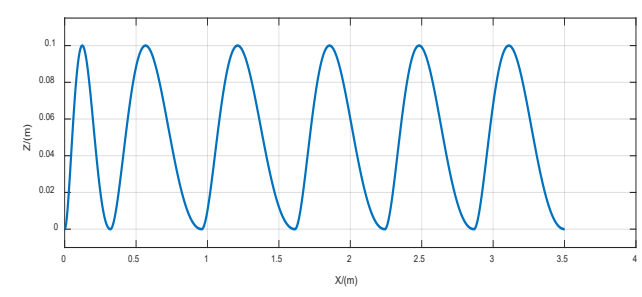

Figure 11. Swing Foot trajectory
Then the exoskeleton gait has been adjusted online by using the balance gait adaptive regulation in order to implement a better ZMP stability. Considering the continuous walking procedure, the left and right leg joint motion have been provided by the exoskeleton kinematic analysis. The planning results and PID controller tracking results are shown in Figure 12.
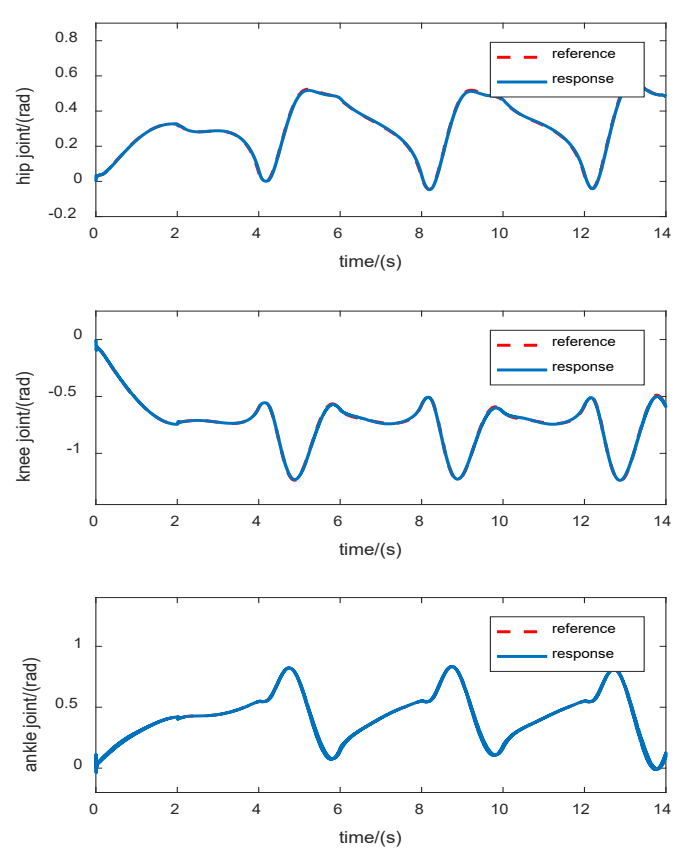

(a) The hip, knee and ankle joint results of right leg
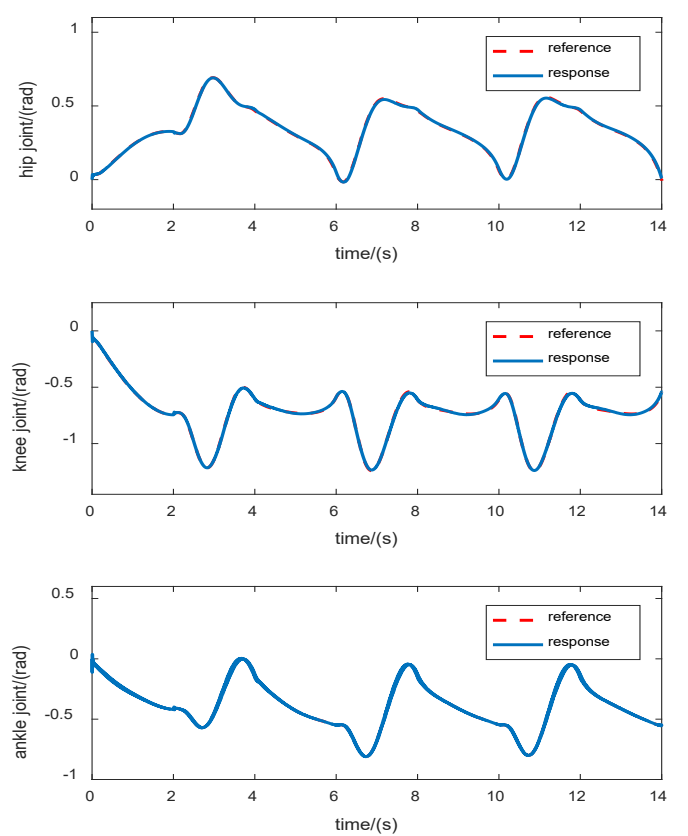

(b) The hip, knee and ankle joint results of left leg

Figure 12. The planning joints and controlling results

https://www.sic.ici.ro 


\subsection{ZMP Balance Simulation Results}

Based on the planning joint results, the walking ZMP results could be obtained, as the element needed to evaluate the balance gait patterns. Here the multibody exoskeleton ZMP results in the proposed online balance gait generation method are compared with those of the conventional method i.e. without online balance gait regulation in the motion procedure from $8 \mathrm{~s}$ to $13 \mathrm{~s}$ as shown in Figure 13.

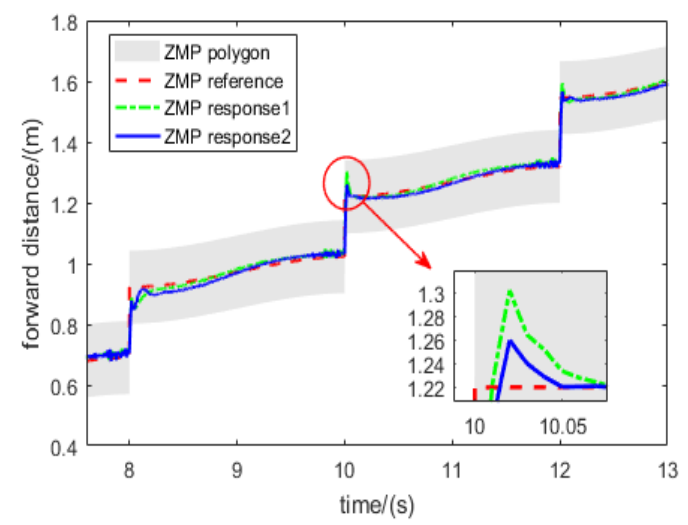

Figure 13. Exoskeleton ZMP simulation result

From Figure 13, the ZMP response1 is generated by conventional method, while ZMP response 2 is generated by the proposed online balance gait generation method respectively. The two ZMP response trajectories that are in the range of the ZMP polygon can be obtained, which guarantees the stable walking. Here, it is assumed that ZMP supporting polygons are set at $+0.12 \mathrm{~m}$ and $-0.12 \mathrm{~m}$ from the center of sole in forward direction. Once the ZMP error becomes large, the proposed method could prevent the ZMP error to increase by comparing to the conventional method.

The unbalance gait usually occurs at DSP, in order to illustrate the balance gait evaluation, the ZMP margin result at about 0 s is shown in the following Table 4 . The number indicates that the minimum distance between sole bounds and multibody exoskeleton ZMP position in the whole walking procedure, at about 10 s has been obtained by the difference between the ZMP response and ZMP stable polygon.The larger obtained number indicates a better walking stability. By contrast, the negative number represents the unstable walking. The referred Table 4 illustrates that the stability is improved, and its effectiveness of online balance gait generation method is demonstrated.

Table 4. Compared ZMP margin results at $10 \mathrm{~s}$

\begin{tabular}{cc}
\hline Compared methods & ZMP margin $(\mathrm{cm})$ \\
\hline $\begin{array}{c}\text { Conventional Method } \\
\text { Online balance gait } \\
\text { generation Method }\end{array}$ & 3.708 \\
\hline
\end{tabular}

\section{Conclusion and Future Work}

In this paper, the online balance gait generation strategy based on human-gait oscillators learning for a walking assistance lower limb rehabilitation exoskeleton has been researched. The mild hemiplegia patients' previous heathy swing foot trajectory characteristics have been utilized for the next exoskeleton motion generation. This proposed method consists of a gait planning strategy and a gait control strategy. In the gait planning strategy, iDE AHopf oscillators have been proposed to learn and model the heathy swing foot trajectory and the dynamic step length estimation method has been utilized to adapt the human motion intension. In the gait control strategy, the online balance gait adaption theory has been proposed to regulate the gait in order to overcome the unbalance gait patterns. From the simulation results, it can be noticed that the proposed method could learn and modify the patients' foot trajectory, estimate adaptively the step length with the patient's CoG position, and improve the walking exoskeleton ZMP margin from $3.71 \mathrm{~cm}$ to $7.984 \mathrm{~cm}$. The final results show that the effectiveness of the gait strategy is verified according to the gait generation results and ZMP stable margin simulation results. A future work will focus upon an improved ZMP correction method especially on its capacity to face the ground disturbances. Other tracking control methods will also be studied.

\section{Acknowledgments}

This work was partially supported by the National Natural Science Foundation of China (61773212), by International Science \& Technology Cooperation Program of China (2015DFA01710), and by the Natural Science Foundation of Jiangsu Province (BK20170094). 


\section{REFERENCES}

Ahmed, A. I., Cheng, H., Liangwei, Z., Omer, M. \& Lin, X. (2017). On-line walking speed control in human-powered exoskeleton systems based on dual reaction force sensors, Journal of Intelligent \& Robotic Systems, 87(1), 59-80.

Bajrami, X., Dermaku, A., Likaj, R., Demaku, N., Kikaj, A., Maloku, S. \& Kikaj, D. (2016). Trajectory planning and inverse kinematics solver for real biped robot with 10 DOF-s, IFAC-PapersOnLine, 49(29), 88-93.

Cannell, J., Jovic, E., Rathjen, A., Lane, K., Tyson, A., Callisaya, M. L., Smith, S. T., Ahuja, K. D. \& Bird, M. (2018). The efficacy of interactive, motion capturebased rehabilitation on functional outcomes in an inpatient stroke population: a randomized controlled trial, Clinical Rehabilitation, 32(2), 191-200.

Chafroud, N., Haddad, N. K. \& Braiek, N. B. (2017).

Stable walking of humanoid robot based on a liquid level model. In International Conference on Advanced Systems and Electrical Technologies (IC_ASET) (pp. 360-365).

Chen, Q., Cheng, H., Yue, C., Huang, R. \& Guo, H. (2017). Step length adaptation for walking assistance. In 2017 IEEE International Conference on Mechatronics and Automation (ICMA) (pp. 644-650).

Chen, Q., Cheng, H., Yue, C., Huang, R. \& Guo, H. (2018). Dynamic balance gait for walking assistance exoskeleton, Applied Bionics and Biomechanics, 10 pages. Article ID 7847014. DOI: $10.1155 / 2018 / 7847014$.

Ding, J., Xiao, X., Wang, Y. \& Xu, B. (2015). Preview control with an angle adjustment strategy for robust real-time biped walking pattern generation. In International Conference on Intelligent Robotics and Applications (ICIRA) (pp. 321-332).

Dumitru, N., Copilusi, P. C. \& Ciurezu, L. (2018). Cam mechanism design for a human leg exoskeleton, Applied Mechanics and Materials, 880, 95-100.

Esquenazi, A., Talaty, M. \& Jayaraman, A. (2017). Powered exoskeletons for walking assistance in persons with central nervous system injuries: a narrative review, $P M \& R, 9(1), 46-62$.

Farzadpour, F. \& Danesh, M. (2012). GA based trajectory generation for a 7-DOF biped robot by considering feet rotation during double support phase, Advanced Materials Research, 463, 1252-1255.

Fu, G. P., Yang, Y. M. \& Huang, C. L. (2011). Walking pattern optimization based on particle swarm optimization for biped robot, Journal of Huazhong University of Science and Technology (Natural Science Edition), 39, 355-358.
Han, S., Wang, H. \& Tian, Y. (2018). Model-free based adaptive nonsingular fast terminal sliding mode control with time-delay estimation for a 12 DOF multi-functional lower limb exoskeleton, Advances in Engineering Software, 119, 38-47.

Hamaya, M., Matsubara, T., Noda, T., Teramae, T. \& Morimoto, J. (2017). Learning assistive strategies for exoskeleton robots from user-robot physical interaction, Pattern Recognition Letters, 99, 67-76.

Huang, R., Cheng, H., Guo, H., Lin, X. \& Zhang, J. (2018). Hierarchical learning control with physical human-exoskeleton interaction, Information Sciences, 432, 584-595.

Kajita, S., Kanehiro, F., Kaneko, K., Fujiwara, K., Harada, K., Yokoi, K. \& Hirukawa, H. (2003). Biped walking pattern generation by using preview control of zero-moment point. In 2003 IEEE International Conference on Robotics and Automation (pp. 1620-1626).

van Kammen, K., Boonstra, A. M., Woude, L. H., Reinders-Messelink, H. A. \& Otter, R. D. (2016). The combined effects of guidance force, bodyweight support and gait speed on muscle activity during able-bodied walking in the Lokomat, Clinical Biomechanics, 36, 65-73.

Kulvicius, T., Biehl, M., Aein, M. J., Tamosiunaite, M. \& Wörgötter, F. (2013). Interaction learning for dynamic movement primitives used in cooperative robotic tasks, Robotics and Autonomous Systems, 61(12), 1450-1459.

Liu, H., Jia, W. \& Bi, L. (2017). Hopf oscillator based adaptive locomotion control for a bionic quadruped robot. In IEEE International Conference on Mechatronics and Automation (pp. 949-954).

Long, Y., Du, Z. J., Wang, W. D. \& Dong, W. (2018). Human motion intent learning based motion assistance control for a wearable exoskeleton, Robotics and Computer-Integrated Manufacturing, 49, 317-327.

Mondal, S., Nandy, A., Verma, C., Shukla, S., Saxena, N., Chakraborty, P. \& Nandi, G. C. (2011). Modeling a central pattern generator to generate the biped locomotion of a bipedal robot using Rayleigh oscillators. In International Conference on Contemporary Computing - IC3 (pp. 289-300).

Qin, A. K., Huang, V. L. \& Suganthan, P. N. (2009). Differential evolution algorithm with strategy adaptation for global numerical optimization, IEEE Transactions on Evolutionary Computation, 13(2), 398-417.

Righetti, L., Buchli, J. \& Ijspeert, A. J. (2006). Dynamic hebbian learning in adaptive frequency 
oscillators, Physica D: Nonlinear Phenomena, 216(2), 269-281.

Righetti, L. \& Ijspeert, A. J. (2006). Programmable central pattern generators: an application to biped locomotion control. In Proceedings of 2006 IEEE International Conference on Robotics and Automation (ICRA) (pp. 1585-1590).

Sato, T., Sakaino, S., Ohashi, E. \& Ohnishi, K. (2011). Walking trajectory planning on stairs using virtual slope for biped robots, IEEE Transactions on Industrial Electronics, 58(4), 1385-1396.

Sato, T., Sakaino, S. \& Ohnishi, K. (2008). Parameter design for ZMP disturbance observer of biped robot. In $34^{\text {th }}$ Annual Conference of IEEE Industrial Electronics, (pp. 1650-1655).

Seleem, I. A. \& Assal, S. F. (2016). A neuro fuzzybased gait trajectory generator for a biped robot using Kinect data. In $3^{\text {rd }}$ International Conference on Information Science and Control Engineering (ICISCE) (pp. 763-768).

Sharma, V. P., Choudhary, H. R., Kumar, S. K. \& Choudhary, V. (2015). A modified DE: Population or generation based levy flight differential evolution (PGLFDE). In 2015 International Conference on Futuristic Trends on Computational Analysis and Knowledge Management (ABLAZE) (pp. 704-710).

Simbolotti, C., Molteni, F., Guanziroli, E., Iacovelli, C., Padua, L., Cicetti, S., Caloi, M. \& Caliandro, P. (2016). Gait training with Ekso in ischemic chronic stroke patients: Effects on the timing of muscle activation and metabolic activation of the prefrontal cortex, Gait \& Posture, 49(1), 27-28.

Vallery, H., Asseldonk, E. V., Buss, M. \& Kooij, H. V. (2009). Reference trajectory generation for rehabilitation robots: complementary limb motion estimation, IEEE Transactions on Neural Systems and Rehabilitation Engineering, 17(1), 23-30.

Wang, X., Li, X., Wang, J., Fang, X. \& Zhu, X. (2016). Data-driven model-free adaptive sliding mode control for the multi degree-of-freedom robotic exoskeleton, Information Sciences, 327, 246-257.

Wu, N., Chew, C., Poo, A. N. \& Li, R. (2013). Moving Horizontal Reference Map for Bipedal Robot Walking Over Uneven Terrain, IFAC Proceedings Volumes, $46(5), 577-582$

Zeilig, G., Weingarden, H., Zwecker, M., Dudkiewicz, I., Bloch, A. \& Esquenazi, A. (2012). Safety and tolerance of the ReWalk ${ }^{\mathrm{TM}}$ exoskeleton suit for ambulation by people with complete spinal cord injury: A pilot study, The Journal of Spinal Cord Medicine, 35(2), 96-101.

Zhan, J., Xiong, R. \& Sun, Y. (2013). A ZMP and CoM online replanning method for stable walking of bipedal robots. In $25^{\text {th }}$ Chinese Control and Decision Conference (CCDC) (pp. 2605-2611).

Zhang, J., Fiers, P., Witte, K. A., Jackson, R. W., Poggensee, K. L., Atkeson, C. G. \& Collins, S. H. (2017). Human-in-the-loop optimization of exoskeleton assistance during walking, Science, 356(6344), 1280-1284 